\title{
Development of a Measurement System for Spectrum Awareness (Invited paper)
}

\author{
Kenta Umebayashi†, and Samuli Tiiro \\ Tokyo University of Agriculture and Technology, \\ 2-24-16, Nakacho, Koganei-shi, Tokyo, Japan 184-8588 \\ Email: ume_k@cc.tuat.ac.jp $\dagger$
}

\author{
Janne J. Lehtomäki \\ Centre for Wireless Communications, University of Oulu \\ P. O. BOX 4500 FIN-90014 University of Oulu, Finland
}

\begin{abstract}
Dynamic spectrum access (DSA) is an attractive approach to solve the spectrum scarcity problem. Among DSA techniques, spectrum overlay is an approach where the spectrum licensed to a primary user (PU) is shared by a secondary user (SU) while protecting the $\mathrm{PU}$ from the interference caused by $\mathrm{SU}$ spectrum reuse. In the case where $P U$ traffic is dynamic, spectrum sharing is difficult as satisfying requirements of spectrum sensing, the challenging accuracy, quickness and low cost requirements in practice, is difficult. For this issue, we propose a new concept of smart spectrum access (SSA) where useful information related to PU spectrum utilization is used to achieve not only the above requirements but also more efficient spectrum utilization. We also show an approach to realize practical SSA and it consists of a spectrum awareness system (SAS) and a dynamic spectrum access system (DSAS). The main role of the SAS is to provide useful information to the DSAS. The information can be obtained by the SAS through spectrum utilization measurement and analysis of the measurement data. In this paper, we present a framework for smart spectrum access and discuss the challenges of this approach. In addition, we report some of the experimental results related to SAS.
\end{abstract}

\section{INTRODUCTION}

To resolve the spectrum scarcity problem, dynamic spectrum access (DSA) using cognitive radio techniques employed by the unlicensed user (secondary user: SU) on the spectrum reserved for the licensed user (primary user: PU) has been investigated [1]. Spectrum overlay is one of the possible DSA techniques where the goal is to achieve more flexible and efficient spectrum management [2]. In spectrum overlay based DSA, the SU can utilize spectrum left vacant by the PU as long as it will not cause any harmful interference. In order to enable DSA, techniques for understanding current spectrum state at given time/location, such as spectrum sensing [3] and geolocation database, have been investigated.

In TV white space (TVWS) which is one of DSA approaches, the spectrum sharing can be enabled by using a geolocation database [4]. Spectrum sharing based on the geolocation database is suitable due to the static nature of the TV broadcast traffic. On the other hand, in mobile wireless systems where the traffic is sporadic and the terminals can move around this is not possible and the SU has to rely to other means of obtaining the spectrum state.

However, in practice, the requirements for spectrum sensing are very challenging. Specifically, very accurate PU signal detection performance is required. For example, detection probability of higher than $90 \%$ at low SNR region, such as less than $-10 \mathrm{~dB}$, has been suggested [5]. Another important issue is fast detection, i.e., the SUs are expected to detect the presence of PU signals as fast as possible. Achieving these goals within the limitations of computational requirements and cost requirements is very challenging. The first two requirements, accuracy and quickness, are for protecting PUs properly and the last one is to achieve practical SU terminal.

To enable spectrum sharing in the case of dynamic PU, awareness of the instantaneous state of the spectrum (occupied/vacant) with the above requirements is significant issue. For this issue, we propose a concept of smart spectrum access (SSA). In a typical DSA approach, there are two important points; the first one is how to share and utilize the spectrum among heterogeneous wireless systems [2] and second one is how to find available spectrum. On the other hand, SSA exploits information about the PU spectrum utilization which has potential to achieve not only the requirements of spectrum sensing but also to provide other benefits for DSA. In fact, current state of the spectrum, i.e. vacancy or occupancy, is a target of spectrum sensing. In SSA, any useful information such as statistical information of the spectrum utilization can be target. For example, it has been shown that knowing the channel occupancy rate [6] can improve spectrum sensing performance [7]. In [8], statistical channel utilization model was also used to improve spectrum sensing performance. Channel occupancy rate is also useful to achieve efficient MAC protocol design and spectrum management method as has been demonstrated in [9]-[11].

In fact, there are many related works where statistical information in terms of the spectrum utilization is used to enhance the performance of DSA. In [12], several key techniques and theory for opportunistic spectrum access have been introduced for example game theory, Markovian decision process, optimal stopping theory and multi-armed bandit problem. It can be summarized that there is still a common significant issue; how to obtain the statistical information. In fact, this is related to feasibility of DSA such as the issue in spectrum sensing. In SSA, we will consider this issue carefully for achieving practical SSA and this point is the most significant difference compared to traditional DSA.

In SSA, the following key issues have to be considered:

1) How much gain, for example in terms of spectrum uti- 
lization efficiency and spectrum awareness performance, can be obtained by exploiting the spectrum utilization statistics?

2) How can we obtain this information as efficiently as possible?

3) How does the whole system and processes for obtaining the information and providing it to the DSA, have to be designed?

In the past works regarding the DSA, the first issue has been considered to a certain extent but still the effect of estimation error of the utilization statistics and required cost to obtain the statistical information have not been investigated deeply. The second issue is related to the actual implementation issues of the statistics acquisition such as computational costs and feasibility. To obtain the statistics of spectrum utilization by the PUs, long duration, wide band and broad area spectrum measurements are required. This obviously leads to significant burden in the SUs. The third issue is related to the earlier issues. Specifically, SSA system can be implemented as a network which we have to design and allocate the roles to the entities of the network to achieve an effective SSA system.

SSA has two important roles, spectrum awareness and spectrum access for actual wireless communication services. In our approach, the roles are assigned to different systems, namely spectrum awareness systems (SAS) and dynamic spectrum access systems (DSAS), respectively. This approach can lessen the burden of spectrum measurement of the SUs in DSAS. However, this opens up a new challenge which is the development of an efficient SAS.

The main role of SAS is to obtain and provide useful statistics (such as duty cycle, channel occupancy rate [7] as well as statistics of the idle and busy periods [13]) related to spectrum utilization by PUs to the DSAS based on the spectrum awareness process. By using this information in the DSAS, not only spectrum sensing but also prediction of spectrum utilization and spectrum resource management can be enhanced. In fact, this approach is useful not only for the spectrum overlay DSA, such as cognitive radio systems, but also other spectrum sharing systems such as 802.11 MAC protocol based on wireless local area networks, wireless systems in Industry-Science-Medical (ISM) bands and interoperator spectrum sharing which have been investigated for future wireless systems such as 5G. [14].

This paper is divided in two parts. The first part, Section II, describes the concept of SSA using SAS and DSAS. We also discuss the issues related to the design of efficient SAS and DSAS.

In the second part, Section III, we present a prototype of SAS that we have developed. We have investigated the design issue of spectrum measurement for duty cycle estimation for which we provide numerical evaluation results obtained by computer simulations and real spectrum measurement experiments.

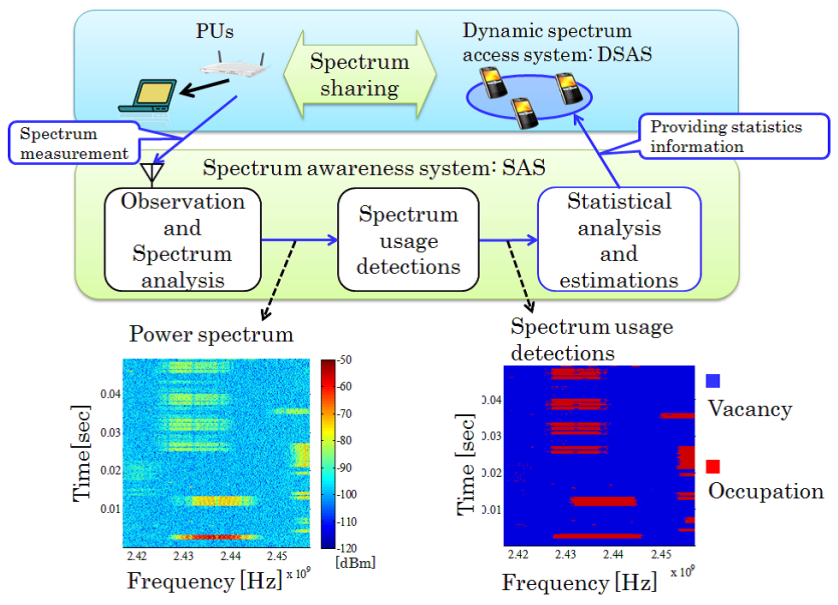

Fig. 1: Smart spectrum access for spectrum overlay scenario.

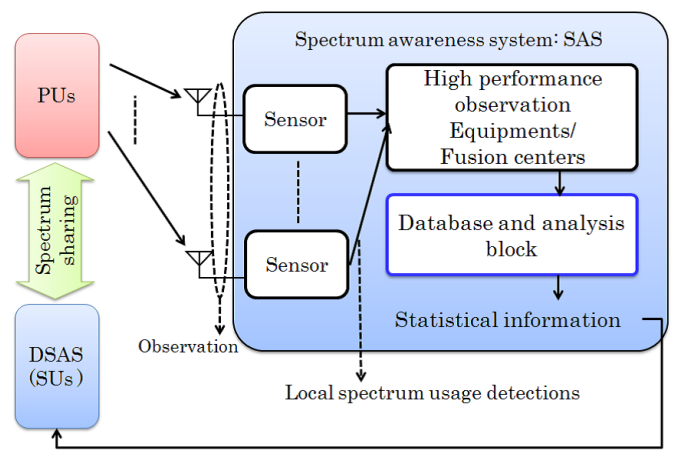

Fig. 2: Wireless network implemented SAS.

\section{SMART SPECTRUM ACCESS}

We introduce the envisioned SSA concept which is shown in Fig. 1. In this example, we assume spectrum overlay scenario, i.e., there are PUs who own the spectrum and SUs who can opportunistically access the spectrum as long as the PU protection constraint is satisfied. In addition, there is an SSA system which consists of DSAS and SAS as described earlier. The SSA is divided into two separate systems each responsible for different functionalities which has the benefit of simplifying the subsystem design and lessening the computational load.

SAS is dedicated for spectrum awareness. The essential functions of the SAS are observation, spectrum analysis (e.g. power spectrum via fast Fourier transform), spectrum usage detection, and analysis process for obtaining the spectrum utilization statistics. These functions are illustrated in the block diagram in Fig. 1. The observation block includes the part from the RF frontend to the analog-to-digital-converter (ADC) which is responsible for obtaining the I-Q baseband samples. Spectrum analysis process then converts the measured IQ samples into power spectral density (PSD) information which is more practical for later analysis. Based on the PSD, spectrum usage detection block provides measured spectrum utilization data in a two-dimensional time-frequency grid. 
Finally, statistical information, such as duty cycle and statistics of the idle and busy periods, can be estimated based on the spectrum usage detection result.

SAS is expected to cover wide area as well as observe a wide band of spectrum for sufficiently long time. In order to cover a wide enough area, it is necessary to deploy a large number of observation equipment (OE). Deployment of high performance OEs commonly used in spectrum measurement is not feasible due to high costs but on the other hand, cheaper OEs, such as sensor in wireless sensor networks, might not be able to satisfy the required measurement accuracy.

In fact, the SAS has to be implemented to interface with a wireless network and an example of such implementation shown in Fig. 2. In our approach, SAS consists of a small number of high performance OEs and many low-cost OEs, i.e. sensors. The high performance OEs also serve as a fusion center and control station for the sensors which are within the respective coverage area. To achieve accurate measurement results with the sensors, one approach to enhance the accuracy is cooperative measurement with multiple sensors. This is similar to cooperative spectrum sensing which can achieve improved sensing performance by exploiting spatial diversity gain [3], [15]. However, additional time is required to gather the spectrum usage/utilization information within the SSA network/system coverage. In spectrum sensing, the time cost is not negligible since spectrum sensing has to know state of spectrum instantaneously. On the other hand, in spectrum usage measurement, the time cost is not a critical issue.

The SSA system has to be designed properly by considering all the different functions jointly. There can be multiple ways to obtain the information, thus, we need to consider for example the computational cost at each sensor, required burden of information gathering in cooperative measurement, and how much the performance of DSAS can be improved when designing the SSA. For example, the type of the estimated statistical information obtained by the SAS determines the achievable performance gain that can be provided to the DSAS as well as the required computational cost and load at each sensor. In our approach, the functions of observation and spectrum usage detection are implemented at the sensor level. This can reduce the burden of communication between sensor and high performance $\mathrm{OE}$ since the spectrum usage detection is binary information. However, this requires us to develop reliable, low complexity signal detection methods.

In the database and analysis block, the measured spectrum utilization data is at first stored in a two-dimensional timefrequency grid. This data can be later used to analyze the statistical characteristics such as duty cycle, channel occupancy rate, and statistics of the idle and busy periods of the spectrum utilization. The statistical data is stored in the database for the required time and can be update according to newer measurement results. Finally SAS provide the statistical information to DSAS to enhance the performance of the DSAS.

\section{PROTOTYPE OF SPECTRUM AWARENESS SYSTEM}

In this section, we present a spectrum measurement prototype which corresponds to a possible implementation of the SAS. Specifically, obviously there is other approaches to realize SAS. So far, the developed prototype has been investigated to provide accurate spectrum usage detection by using Welch FFT based signal detection. In this prototype, all of function blocks in Fig. 2 are involved but we have mainly investigated signal processing parts in spectrum analysis and spectrum usage detection.

\section{A. Issue of threshold setting in spectrum usage detection}

In most of the spectrum measurements [4], [16], energy detector (ED) with threshold is used to detect PU spectrum utilization in the considered frequency bins. ED is simple and does not require any prior information about the PU signal [17], however the detection performance depends on the threshold setting [18], [19]. There are several ways to set the threshold and the most appropriate approach is constant false alarm rate (CFAR). In this case, noise floor (NF) estimation is an important issue for the threshold setting, however most of the previous works have not considered this issue deeply but instead assumed that the NF is known.

In [20], [21], median filtered FCME with a correction factor $\beta$ (MED-FCME- $\beta$ ) has been proposed and this algorithm can estimate NF properly. Thus, the threshold can be set according to target false alarm probability. The median filter is used to avoid the effect of large outliers and $\beta$ is used to compensate the effect of biased estimation error.

We have revealed that the NF estimation performance of the MED-FCME- $\beta$ deteriorates in the region where SNR $\gamma$ is around zero. Similar problem has been observed in several works for example [22], [23]. This degradation is due to frequency fluctuation caused by randomness of data symbols. For this issue, we used Welch FFT in MED-FCME- $\beta$ and this method is denoted by MED-FCME-Welch [24]. In [25]-[27], Welch's method has been employed for signal detection. In [24], the benefits of Welch FFT in the NF and duty cycle (DC) estimations have been shown through computer simulation based numerical evaluations. We will show the benefits by experimental evaluations with a prototype system of SAS system and this can provide validity of the prototype system. estimation and DC estimation.

\section{B. System model}

Block diagram of the prototype system with single $\mathrm{OE}$ is shown in Fig. 3. Cooperative measurement has not been implemented yet and this is a future issue. The target frequency band of our measurement system is $2.4 \mathrm{G} \mathrm{Hz}$ industryscience-medical (ISM) band. This is because that in this band variegated and dynamical spectrum usages can be expected and we would like to evaluate our measurement system in such situations.

In this prototype system, there are a real-time spectrum analyzer (RSA6100A), high capacity hard disk, and two computers, control computer (PC 1) and signal processing 


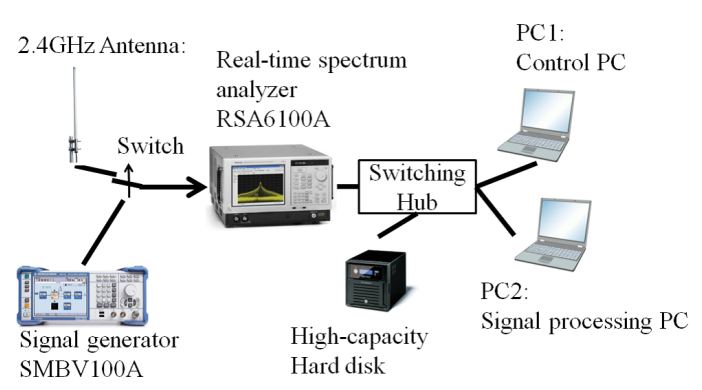

Fig. 3: Prototype system of spectrum measurement.

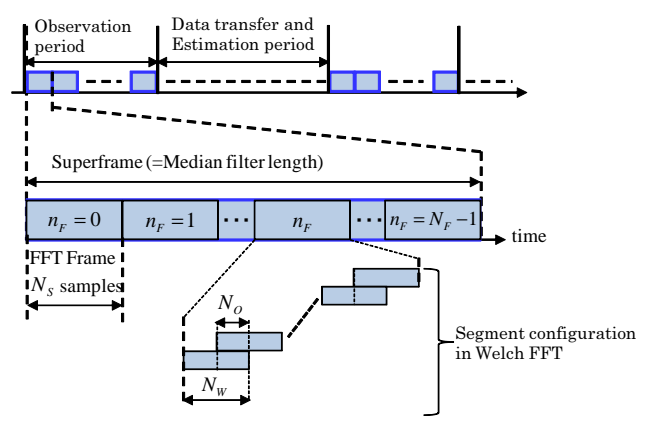

Fig. 4: Time frame configuration of spectrum usage measurement in the SAS system.

computer (PC 2). The role of PC 1 is providing a trigger signal in terms of spectrum measurement schedule presented by time frame as shown in Fig. 4, and information of spectrum measurement specifications to the real-time spectrum analyzer. Based on the instruction by PC 1, the real-time spectrum analyzer obtains I-Q samples for the studied frequency band during the observation period and stores the obtained data to the hard disk during data transfer and estimation period. This means the role of observation is implemented in the real-time spectrum analyzer.

Spectrum analysis, spectrum usage detection and DC estimation are implemented in the PC 2. During data transfer and estimation period, PC 2 obtains a stored data corresponding to observation during one observation period. In PC 2, all processes shown in Fig. 5 are performed in Fig. 5 according to time frames, superframe and FFT frame. These configurations of superframe, and FFT frame represent process units of NF estimation, and Welch FFT, respectively. The observation period is composed hierarchically and one superframe consists of $N_{F}$ frames.

Now we focus on data analysis in the analysis computer in the $n_{F}$ th frame. The block diagram of the data analysis part is shown in Fig. 5. During one FFT frame, the OE obtains observed equivalent baseband signal $y[n](n=$ $\left.n_{F} N_{S}, n_{F} N_{S}+1, \cdots n_{F} N_{S}+N_{S}-1\right)$ with sampling rate $f_{s} \mathrm{~Hz}$ and the sampled complex signal is given by

$$
y[n]=x[n]+z[n]
$$

where $x[n]$ represents PU signal component, $z[n]$ represent

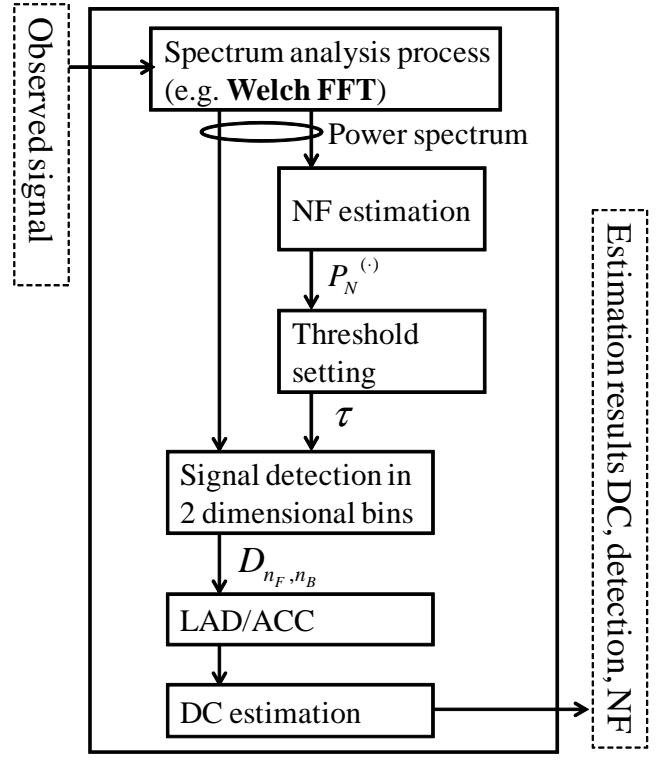

Fig. 5: Block diagram of spectrum analysis, spectrum usage detection, and DC estimation.

noise component which is circularly symmetric normal complex random variable with zero mean and unit variance. SNR is defined by $\gamma=E\left[|x[n]|^{2}\right] / E\left[|z[n]|^{2}\right]$ and $E[]$ is expectation operator. Without loss of generality $N_{S}$ is assumed to be power of two. In the Welch FFT, $N_{S}$ samples are divided into $N_{\text {seg }}$ sample groups where each group consists of $N_{W}$ samples. The configuration is shown in Fig. 4. In the $n_{\text {seg }}$ th segment, observed time complex samples $y_{n_{\text {seg }}}[n]\left(n=0,1, \cdots, N_{W}-1\right)$ are given by

$$
y_{n_{\text {seg }}}[n]=y\left[n+\left(n_{\text {seg }}-1\right) N_{W} / 2\right],
$$

without loss of generality we assume $N_{W}$ is even number and there is overlapped samples between neighboring sample groups, i.e. the number of overlapped samples $N_{O}$ is set to $N_{W} / 2=N_{O}$. In this case, $N_{S}, N_{s e g}$ and $N_{W}$ satisfies the following equation:

$$
N_{\text {seg }}=\left(2 N_{S}\right) /\left(N_{W}\right)-1
$$

where $N_{W}$ is assumed to be divisor of $2 N_{S}$.

Then, the discrete power spectrum density is given by

$$
P_{n_{\mathrm{seg}}, c_{\mathrm{seg}}}^{(\mathrm{seg})}=\left|\frac{1}{\sqrt{N_{W}}} \sum_{k=0}^{N_{W}-1}\left(h_{h}[k] y_{n_{\mathrm{seg}}}[k] e^{-j 2 \pi c_{\text {seg }} k}\right)\right|^{2}
$$

where $c_{\text {seg }}$ is the index number of the frequency bin and $h_{h}[k]$ is hamming window. In this case, the power spectrum density based on Welch FFT is given by

$$
P_{n_{F}, c_{\text {seg }}}^{(\text {Welch })}=\frac{1}{N_{\text {seg }}} \sum_{n_{\text {seg }}=0}^{N_{\text {seg }}-1} P_{n_{\mathrm{seg}}, c_{\mathrm{seg}}}^{(\mathrm{seg})}
$$

The averaging process in the the above equation can suppress the frequency fluctuation. Specifically, each spectrum 
in segment $P_{n_{\mathrm{seg}}, c_{\mathrm{seg}}}^{(\mathrm{seg})}$ has independent frequency selectivity characteristic and the averaging leads to nearly flat spectrum.

In the NF estimation block, NF estimation is performed every time frame and the estimated noise power is denoted by $P_{N}\left(n_{F}\right)$. To avoid effect of outliers of frequency fluctuation, median filter is used. The details of the NF estimation methods is shown in [24].

In the threshold setting block, the threshold $\tau$ is set based on the target false alarm rate $\dot{P}_{\mathrm{FA}}$. Specifically $\tau$ has to satisfy the following equation

$$
\dot{P}_{\mathrm{FA}}=\operatorname{Pr}\left(P_{n_{F}, n_{B}}>\tau \mid H_{0}\right),
$$

where $\operatorname{Pr}()$ indicates a probability for an event.

In the signal detection block, based on the threshold $\tau$, signal occupancy is detected at two dimensional bin $\left(n_{F}, n_{B}\right)$ as

$$
D_{n_{F}, n_{B}}= \begin{cases}1 & \left(P_{n_{F}, n_{B}}>\tau\right) \\ 0 & \text { (otherwise) }\end{cases}
$$

Although median filter can provide accurate NF estimation, misdetection of the PU channel usage would still be possible with ED. For this issue, localization algorithm based on double-thresholding (LAD) with adjacent cluster combining (ACC), LAD-ACC, is used in [21].

LAD-ACC algorithm composed of LAD process and ACC process, and details are shown for example in [21], [24]. In the LAD, two thresholds, low threshold $\tau_{\mathrm{L}}$ and high threshold $\tau_{\mathrm{H}}$, are used and they are set based on target clean sample rejection rate (CSRR), $\dot{P}_{\mathrm{FA}, \mathrm{L}}$ and $\dot{P}_{\mathrm{FA}, \mathrm{H}}$, respectively. Using the low threshold is possible to achieve high sensitivity for detecting low level signal while less false alarm probability can be achieved by the high threshold. In addition, ACC process can further compensate the miss detection.

The output of ACC based on $D_{n_{F}, n_{B}}$ is denoted by $A_{n_{F}, n_{B}}$ and $\mathrm{DC}$ for a bin $n_{B}$ is given by

$$
D C=\frac{1}{n_{B, \max }} \sum_{k=0}^{n_{B, \max }} A_{n_{F}, k},
$$

where $n_{B, \max }$ is the number of observed time samples in a superframe.

\section{Experimental results based on the prototype system}

We evaluate DC estimation performances in terms of MEDFCME- $\beta$ and MED-FCME-Welch. The main difference between two methods is that Welch FFT is used in MED-FCMEWelch, but normal FFT is used in MED-FCME- $\beta$. There are three target probabilities, the first one is CSRR for setting $\dot{P}_{\mathrm{FA}, \mathrm{CSRR}}$ for setting $T_{\mathrm{CME}}$, the second one is $\dot{P}_{\mathrm{FA}, \mathrm{L}}$ for setting $\tau_{L}$, and the third one is $\dot{P}_{\mathrm{FA}, \mathrm{H}}$ for setting $\tau_{H}$. They are set as follows; $\dot{P}_{\mathrm{FA}, \mathrm{CSRR}}=0.01, \dot{P}_{\mathrm{FA}, \mathrm{H}}=4.54 \cdot 10^{-5}$, and $\dot{P}_{\mathrm{FA}, \mathrm{L}}=0.01$. FFT size $N_{S}$ and segment size in Welch FFT $N_{W}$ are set to 1024 and 64 , respectively.

In [24], we have confirmed that MED-FCME-Welch can achieve better DC estimation performance by computer simulation, but now we confirm it with experimental demonstration. The SG transmits data packet based on IEEE $802.11 \mathrm{~g}$ standard with a specified DC to real-time spectrum analyzer (RSA6100A) with a cable. The bandwidth of the transmit signal is around $20 \mathrm{MHz}$ and the observed bandwidth is set to $40 \mathrm{MHz}$. The DC estimation performance as a function of transmit power of the SG is shown in Fig. 6 with different DCs $(0.1,0.52,0.89)$. We can confirm that our proposed method outperforms the MED-FCME- $\beta$. The gain obtained by the MED-FCME-Welch compared to the MED-FCME- $\beta$ is from $12 \mathrm{~dB}$ to $15 \mathrm{~dB}$ and this is obtained by $N_{\text {seg }}=31$ times averaging process, i.e. $10 \log _{10} 31 \approx 14.9 \mathrm{~dB}$.

The location of the experiment is in 4 th floor, building number 5, Tokyo university of agriculture and technology, in Tokyo, Japan. The university is not located in downtown Tokyo, but it is located in small city. In the 4th floor, there is one laboratory which uses the sixth channel (center frequency is $2.437 \mathrm{GHz}$ ) in $2.4 \mathrm{GHz}$ wireless LAN channel with one access point, and the laboratory locates in front of the location of experiment. The signal transmitted by the access point is the most significant in the observed signal.

The result of DC estimation is shown in Fig. 7. DC estimation obtained by MED-FCME-Welch is always slightly larger than that of MED-FCME- $\beta$ while false alarm performances are almost equivalent. This indicates that sensitivity of MED-FCME-Welch is better than that of MED-FCME- $\beta$. In addition, DC during week days is larger than DC in weekend (Saturday and Sunday). This is because number of users (in this case students and staff in the laboratory) during the weekend is less than the number during weekday. In addition, the behaviors of DC during one day in week days have strong correlations and this is also related to the behavioral pattern of the users.

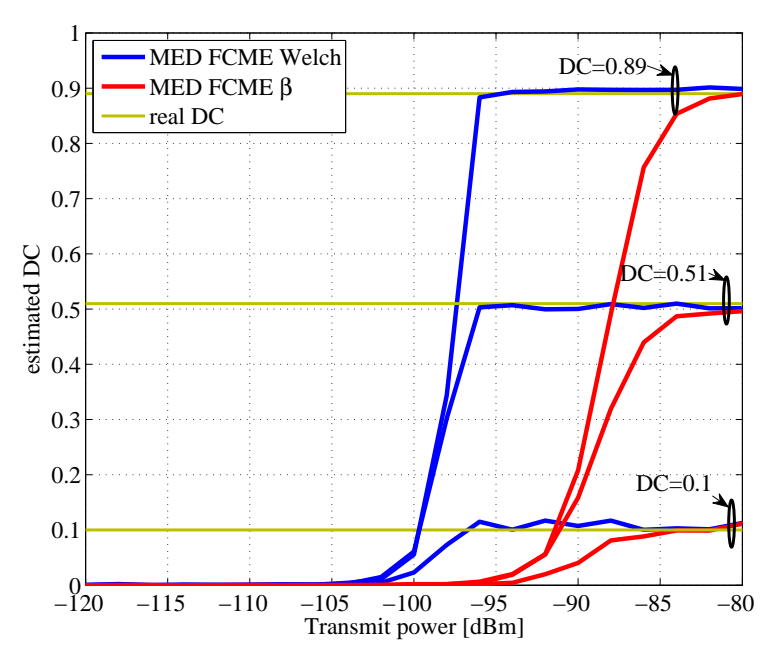

Fig. 6: DC estimation as a function of transmit power at SG (Demonstration experiment).

\section{CONCLUSION}

In this paper, we have shown the idea of smart spectrum access which consists of SAS and DSAS. This approach has a 


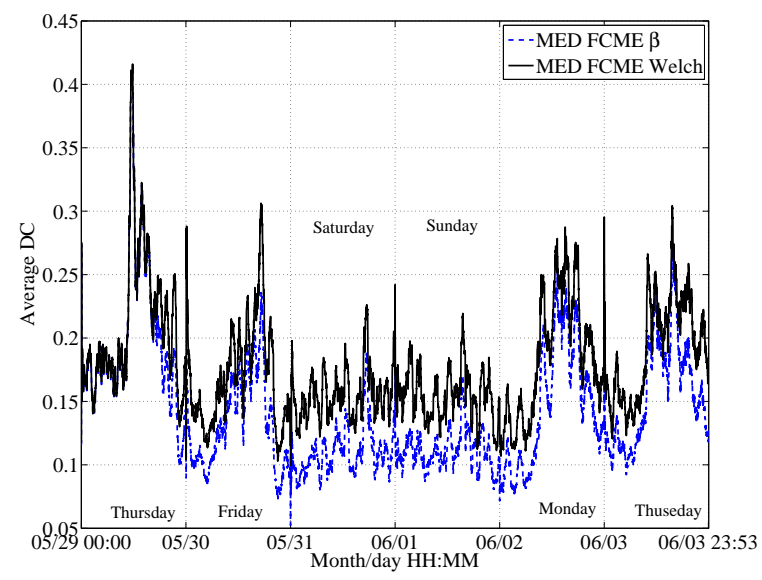

Fig. 7: Average DC estimation for 6 days MED-FCME- $\beta$ and MED-FCME-Welch FFT. Average time is 1 hour.

potential to achieve practical and efficient dynamic spectrum access even if the traffic of PU is dynamic. The main reason of the division of roles, i.e. SAS and DSAS, is to reduce burden per system and terminal. In SAS, cooperative measurement is one of key approaches sine this approach can achieve accurate spectrum awareness due to space diversity while it can maintain low cost OE. However, burden of information gathering in the cooperative measurement is a significant future issue.

We have also shown a prototype system of spectrum awareness system. Experimental results provides a validity of the prototype system.

\section{ACKNOWLEDGMENT}

This research was supported by the Strategic Information and Communications R\&D Promotion Programme (SCOPE).

\section{REFERENCES}

[1] I. F. Akyildiz, W. Y. Lee, M. C. Vuran, and S. Mohanty, "Next generation/dynamic spectrum access/cognitive radio wireless networks: A survey," Computer Networks: The International Journal of Computer and Telecommunications Networking, vol. 50, pp. 2127-2159, Sept. 2006.

[2] Q. Zhao, "A survey of dynamic spectrum access: signal processing, networking, and regulatory policy," IEEE Signal Processing Mag., vol. 24, pp. 79-89, May 2007.

[3] T. Yucek and H. Arslan, "A survey of spectrum sensing algorithms for cognitive radio applications," IEEE Communications Surveys \& Tutorials, vol. 11, pp. 116-130, 2009.

[4] M. Fitch, M. Nekovee, S. Kawade, K. Briggs, and R. MacKenzie, "Wireless service provision in TV white space with cognitive radio technology: A telecom operator's perspective and experience," IEEE Communications Magazine, vol. 49, no. 3, pp. 64-73, 2011

[5] FCC, "Second report and order and memorandum opinion and order: in the matter of unlicensed operation in the TV broadcast bands," FCC, Tech. Rep. Doc. 08-260, Nov. 2008.

[6] A. D. Spaulding and G. H. Hagn, "On the definition and estimation of spectrum occupancy," IEEE Trans. Electromagn. Compat., vol. EMC-19, no. 3, pp. 269-280, Aug. 1977.

[7] N. Wang, Y. Gao, and X. Zhang, "Adaptive spectrum sensing algorithm under different primary user utilizations," IEEE Communications Letters, vol. 17, no. 9, pp. 1838-1841, 2013.
[8] T. Nguyen, B. L. Mark, and Y. Ephraim, "Spectrum sensing using a hidden bivariate markov model." IEEE Transactions on Wireless Communications, vol. 12, no. 9, pp. 4582-4591, 2013.

[9] D. Zhao and X. Zhou, "Spectrum sensing using prior probability prediction," in Proc. of Wireless Communications, Networking and Mobile Computing (WiCOM), Sep. 2011, pp. 1-4.

[10] J. Vartiainen, M. Höyhtyä, J. Lehtomaki, and T. Braysy, "Priority channel selection based on detection history database," in in Proc. of IEEE Cognitive Radio Oriented Wireless Networks \& Communications (CROWNCOM), June 2010, pp. 1-5.

[11] K. Umebayashi, Y. Suzuki, and J. Lehtomäki, "Dynamic selection of CWmin in cognitive radio networks for protecting IEEE 802.11 primary users," in Proc. CROWNCOM, June 2011, pp. 266-270.

[12] Y. Xu, A. Anpalagan, Q. Wu, L. Shen, Z. Gao, and J. Wang, "Decisiontheoretic distributed channel selection for opportunistic spectrum access: Strategies, challenges and solutions," IEEE Communications Surveys and Tutorials, vol. 15, no. 4, pp. 1689-1713, 2013.

[13] M. Lopez-Benitez and F. Casadevall, "Time-dimension models of spectrum usage for the analysis, design, and simulation of cognitive radio networks." IEEE T. Vehicular Technology, vol. 62, no. 5, pp. 2091-2104, 2013.

[14] J. Luo, J. Eichinger, Z. Zhao, and E. Schulz, "Multi-carrier waveform based flexible inter-operator spectrum sharing for $5 \mathrm{~g}$ systems," in in Proc. of Dynamic Spectrum Access Networks (DYSPAN), Apr. 2014, pp. 449 - 457.

[15] H. Uchiyama, K. Umebayashi, T. Fujii, F. Ono, K. Sakaguchi, Y. Kamiya, and Y. Suzuki, "Study on soft decision based cooperative sensing for cognitive radio networks," IEICE Trans. Commun., vol. E91B, no. 1, pp. 95-101, Jan. 2008.

[16] T. M. Taher, R. B. Bacchus, K. J. Zdunek, and D. A. Roberson, "Longterm spectral occupancy findings in Chicago," in in Proc. of IEEE Symposium on Dynamic Spectrum Access Networks, May 2011, pp. 100107.

[17] H. Urkowitz, "Energy detection of unknown deterministic signals," Proc. IEEE, vol. 55, no. 4, pp. 523-531, Apr. 1967.

[18] A. Sahai, N. Hoven, and R. Tandra, "Some fundamental limits on cognitive radio," in in Forty-second Allerton Conference on Communication, Control, and Computing, 2004.

[19] K. Patil, R. Prasad, and K. Skouby, "A survey of worldwide spectrum occupancy measurement campaigns for cognitive radio," in in Proc. of Devices and Communications (ICDeCom), Feb. 2011, pp. 1-5.

[20] J. J. Lehtomäki, R. Vuohtoniemi, K. Umebayashi, and J.-P. Mäkelä, "Energy detection based estimation of channel occupancy rate with adaptive noise estimation," IEICE Trans. Commun., vol. E95-B, no. 4, pp. 1076-1084, Apr. 2012.

[21] J. J. Lehtomaki, R. Vuohtoniemi, and K. Umebayashi, "On the measurement of duty cycle and channel occupancy rate," IEEE J. Select. Areas Commun., vol. 31, no. 1, pp. 2555 - 2565, Nov. 2013.

[22] J. J. Lehtomäki, J. Vartiainen, M. Juntti, and H. Saarnisaari, "Spectrum sensing with forward methods," in Proc. IEEE Military Communications Conference (MILCOM), Washington DC, Oct. 2006, pp. 23-25.

[23] J. Lehtomaki, M. Juntti, and H. Saarnisaari, "CFAR strategies for channelized radiometer," IEEE Signal Processing Lett., vol. 12, pp. 1316, Jan. 2005.

[24] K. Umebayashi, R. Takagi, N. Ioroi, Y. Suzuki, and J. J. Lehtomaki, "Duty cycle and noise floor estimation with welch fft for spectrum usage measurements," in in Proc. of Cognitive Radio Oriented Wireless Networks and Communications (CROWNCOM), June 2014, pp. 73 - 78.

[25] R. S. Walker, "The detection performance of fft processors for narrowband signals," Defence Research Establishment Atlantic, Tech. Rep., Feb. 1982.

[26] H. Sarvanko, M. Mustonen, A. Hekkala, A. Mammela, M. Matinmikko, and M. Katz, "Cooperative and noncooperative spectrum sensing techniques using welch's periodogram in cognitive radios," in in Proc. of Cognitive Radio and Advanced Spectrum Management international workshop, Feb. 2008, pp. 1-5.

[27] J. Lehtomaki, S. Salmenkaita, J. Vartiainen, J.-P. Makela, R. Vuohtoniemi, and M. Juntti, "Measurement studies of a spectrum sensing algorithm based on double thresholding," in in Proc. of Cognitive Radio and Advanced Spectrum Management international workshop, May 2009, pp. 69-73. 